\title{
TEMAS SELECTOS DE COMERCIO ELECTRÓNICO
}

\author{
Carlos Alberto Rodríguez Garza*, Fabiola de Jesús Mapén Franco*
}

\begin{abstract}
Rodríguez-Garza C.A., Mapén-Franco F.J. Temas selectos de comercio electrónico. Hitos de Ciencias Económico Administrativas 2012;18 (51):73-82.
\end{abstract}

\section{RESUMEN}

Este documento presenta una serie de temas que han salido de estudios realizados sobre el comercio electrónico. Se analizan las ventajas y desventajas resaltando que éstas últimas pueden ser minimizadas. Del mismo modo se presentan algunos aspectos importantes del comercio electrónico como su uso en las PYMES. Asimismo, se descubre que este tipo de comercio no consiste únicamente en contar con una página web, sino que más bien debería seguir un proceso de adopción más que de adaptación.

\begin{abstract}
Rodríguez-Garza C.A., Mapén-Franco F.J. Selected topics of electronic commerce. Hitos de Ciencias Económico Administrativas 2012;18 (51):73-82.
\end{abstract}

\section{ABSTRACT}

This document presents a number of issues that have come from studies on electronic commerce. The advantages and disadvantages are analyzed highlighting that the latter can be minimized. Similarly some important aspects related to the electronic commerce are shown, especially when used in the SMEs. Also it is found out that this type of business must not rely solely on a Web page. On the contrary, it is suggested to follow a process of adaptation rather than adoption.

Palabras clave: Comercio Electrónico, Internet, Nuevas

Key words: Electronic Commerce, Internet, New InforTecnologías de Información y Comunicación (NTIC). mation and Communication Technologies.

DIRECCIÓN PARA RECIBIR CORRESPONDENCIA: Correo electrónico: carlos.rodriguez@ujat.mx; fabiola.mapen@ujat.mx

\section{ROLEGÓMENOS DEL COMERCIO ELECTRÓNICO}

No puede negarse la importancia del comercio en la vida y desarrollo de los pueblos, misma que ha llevado a más de una guerra a través de la historia universal, tanto reales como ficticias; por ello no debe sorprendernos, la importancia que ha tenido en los últimos años el estudio del comercio electrónico.

Aunque el concepto de comercio electrónico incluye muchas formas de intercambio comercial, parece que únicamente se está usando para el comercio a través de Internet. En realidad se debería considerar comercio electrónico, no sólo a este tipo de venta, sino también a la que se hace; por ejemplo, al comprar un logo o una melodía para el teléfono móvil o un partido de fútbol en una plataforma de televisión de pago, incluso extendiendo el significado de electrónico a todo aquello que se compra a través de redes de telecomunicación por medios electrónicos (Pardo, 2004). El comercio electrónico afecta, en mayor o menor medida, a todos los sectores de actividad económica, tanto intrasectorial

\footnotetext{
* Maestro en Administración. Profesor Investigador de la División Académica de Ciencias Económico Administrativas. Universidad Juárez Autónoma de Tabasco.

" Maestra en Administración. Profesora Investigadora de la División Académica de Ciencias Económico Administrativas. Universidad Juárez Autónoma de Tabasco.
}

Fecha de recibido: 5 de abril de 2012 Fecha de aceptación: 7 de mayo de 2012. 
como intersectorialmente, pudiendo establecerse relaciones globales desde el primero al último elemento de las cadenas de valor correspondientes (Castells, 1997).

Al mismo tiempo, hay que analizar la base del comercio electrónico moderno como lo son las Nuevas Tecnologías de Información y Comunicación (NTIC), siendo el más representativo; Internet, al referirnos a las nuevas tecnologías de la información, estamos aludiendo al almacenamiento, procesamiento, recuperación y distribución de la información por medio de procesos microelectrónicos computarizados, lo que se denomina informática, así como de la telemática, que es la organización y transmisión de mensajes computados a través de redes integradas de telecomunicación mediante satélites, la digitalización, la fibra óptica, y las nuevas posibilidades que brindan los instrumentos de comunicación electrónica existentes como son la radio, la televisión, el teléfono, (Jaramillo, 1986).

La diferencia entre TIC (Tecnologías de Información y Comunicación) y NTIC se puede establecer por la llegada de Internet, aunque es bien sabido que los primeros pasos de ésta datan de los años 50 , se desarrollan en los 60 y mejoran en los 70 (sobre todo a finales de 1969 con el nacimiento de ARPANET, el precursor más claro de Internet), es en la década de los 80 cuando se ponen las bases técnicas y los cimientos infraestructurales de Internet que se conocen.

Ahora bien, cuando realmente Internet se desarrolla con gran fuerza, es a partir de los 90 , con el nacimiento de la World Wide Web (un sistema de documentos de hipertexto y/o hipermedios enlazados y accesibles a través de Internet), y para ser más claros, pensamos que el invento más democratizador de Internet fueron los navegadores (Browser), ya que ellos sacaron a ésta, de ser una obscura red académica, a una herramienta global. Con un navegador, un usuario visualiza páginas web que pueden contener texto e imágenes, vídeos u otros contenidos multimedia, navegando a través de ellas usando hiperenlaces. A partir de ese momento, Internet, aporta valor añadido a la informática y a las comunicaciones y, cada vez más, se va conjuntando el término de nuevas tecnologías con el de Tecnologías de la Información y la Comunicación. Probablemente este cambio terminológico obedece a dos factores: por un lado porque las nuevas tecnologías, como todas las cosas nuevas, tienen un carácter perecedero y es evidente que algo que hoy es nuevo no puede seguir siéndolo después de diez o más años. Por otra parte, la aparición de Internet acelera la comunicación y, consecuentemente, el flujo de información generado de manera exponencial, por lo que parece obvio adjetivar aquellas tecnologías (que ya no son nuevas) con términos de actualidad, no perecederos (Información y Comunicación) y así se va suprimiendo del antiguo nombre lo de «nuevas». Esta supresión no ha supuesto la desaparición del término, todavía se sigue utilizando y actualizando, fusionándolo con la nueva terminología. Así hay quien habla de las NTIC; es decir, las Nuevas Tecnologías de la Información y la Comunicación. (Muñoz, 2008).

Un factor que queda reforzado de forma natural mediante el uso del comercio electrónico es el de la universalidad, en el sentido espacial del término, tanto en su aspecto de independencia física respecto al territorio o lugar de trabajo, como en el relativo a la posibilidad de realizar transacciones comerciales de forma global, con cualquier empresa allí donde se encuentre y a cualquier hora (Poster, 1995), (Castells, 1997).

Para ser un medio de comunicación tan joven, Internet ha evolucionado a pasos agigantados; a menos de 15 años del inicio de la comercialización en masa propiciada por la popularidad del web (burbuja punto com), existen ya etapas históricas claramente diferenciables de los paradigmas de Internet, (De la Guardia, 2001).

\section{POSICIONES FRAGMENTADAS ALREDEDOR DEL COMERCIO ELECTRÓNICO}

Existe el debate entre establecer el comercio electrónico como una nueva forma de hacer comercio o solo un nuevo canal, para no entrar en polémica puede distinguirse que en la actualidad, existen básicamente dos tipos de empresas que utilizan las nuevas tecnologías, por un lado, las empresas con presencia física (tradicionales) que entienden las nuevas tecnologías, y en especial Internet, como un nuevo canal de distribución o como lógica extensión de sus negocios, y por otro lado, las empresas puramente digitales, que han estado concebidas para operar en este nuevo entorno, cuyo negocio se basa en la detección de nuevas oportunidades de mercado (Padilla \& Serarols, 2006); 
o sea, no se trata de un solo concepto. Para distinguir a las tiendas que venden en Internet, llamadas «tiendas virtuales», de las tiendas tradicionales o «tiendas reales», a las primeras se les llama de «click» por la operación que se efectúa con el ratón del ordenador y a las segundas de «brick and mortar» expresión inglesa que significa «ladrillos y mortero». Según los expertos, el futuro del comercio minorista es la combinación de ambos sistemas; es decir, vender en el mundo virtual y en el real. La solución, llamada por algunos click and mortar, está por desarrollarse (Pardo, 2004).

Es muy fácil desaprobar el comercio electrónico o negar su uso, también es muy fácil generar expectativas demasiado alentadoras, tiene ventajas y desventajas; por un lado se establecen que los precios son generalmente más bajos que en la empresa tradicional, aunque la política a este respecto es muy variada y depende también del tamaño de la empresa, puede aceptarse como cierta esa premisa, pensado en los ahorros de costos, que trae como consecuencia el uso de Internet, no sólo en la venta, si no también la compra, la logística y otras actividades estratégicas dentro de la empresa; lo que es conocido con el término negocio electrónico (e-business).

Del mismo modo, el comercio electrónico permite mejorar la imagen y las relaciones entre los clientes, la oportunidad de internacionalizarse, mejorar la adquisición de información y conocimiento, libertad y eficacia territorial posibilitada por Internet (Moliní, 2002), se pueden mencionar como los principales beneficios. Frente a las ventajas cuantitativas y cualitativas mencionadas, la lista de desventajas y obstáculos para el comercio electrónico es de menor importancia, aunque no deja de ser numerosa y significativa. Los principales inconvenientes para las empresas son: conexiones en general todavía lentas y con numerosos fallos, aumento de algunos costos informáticos, de personal y de publicidad, no se trata solo de contar con unas páginas Web informáticas, si no exige una nueva estructura organizativa y un nuevo marco de análisis (Conklin \& Tapp, 2000), en muchos casos, la obtención de rendimientos elevados puede requerir una costosa publicidad, la seguridad en la red no está aún del todo lograda, existen ciertas incertidumbres legales y fiscales, todavía muchos consumidores desconfían de la red, en muchas ocasiones los costos de transporte pueden ser superiores al ahorro que representa comprar en Internet, en la actualidad es muy difícil la obtención de financiamiento para la puesta en marcha de una empresa de comercio electrónico y los negocios digitales son fácilmente reproducibles (Moliní, 2002).

Internet está transformando las economías, los mercados y las estructuras de la industria; los productos, los servicios y su circulación; el reparto, los valores y el comportamiento de los consumidores; el mercado de trabajo y sus empleos. Pero el impacto será todavía más grande sobre las sociedades y la política, y por encima de todo, sobre nuestra visión del mundo y sobre nuestro lugar en el mundo.

Al mismo tiempo, nuevas actividades inesperadas van a emerger rápidamente, como la biotecnología. Es muy probable que otras tecnologías emerjan de repente y den lugar a grandes industrias. Estas predicciones se basan en la hipótesis de que la revolución de la información evolucionará como las otras «revoluciones» tecnológicas desde hace 500 años, desde Gutenberg y la revolución de la imprenta en 1455. En particular, la hipótesis es que la revolución de la información seguirá las mismas etapas que la revolución industrial, y es lo que ha ocurrido durante su primer medio siglo.

La revolución de la información está en el mismo punto que la revolución industrial a principios de la década entre 1820-1830.

Unos 40 años después de la primera aplicación industrial, en 1785, de la máquina de vapor de James Watt (creada en 1776) aparece la fábrica de hilados del algodón. El motor de vapor fue para la revolución industrial, lo que la computadora es para la revolución de la información: su detonante, pero ante todo su símbolo. Todo el mundo cree hoy en día que nada en la historia económica ha evolucionado más rápido, o tuvo más impacto, que la revolución de la información. Pero la revolución industrial ha evolucionado igual de rápido y su impacto ha sido probablemente equivalente, si no más importante, por la mecanización de los procedimientos de fabricación, empezando por el textil.

El comercio electrónico es la revolución de la información, lo mismo que supuso el ferrocarril para la 
revolución industrial: un desarrollo sin precedentes, inesperado. Al igual que el ferrocarril hace 170 años, el comercio electrónico está creando un nuevo boom que va a transformar la economía, la sociedad y la política. En la nueva geografía creada por el ferrocarril, la humanidad se ha acostumbrado a las distancias, en la del comercio electrónico se han eliminado. Únicamente hay una economía, sólo hay un mercado. En consecuencia, cada comercio debe volverse competitivo, incluso si sólo fabrica o vende localmente o regionalmente. La competencia ya no es local, no conoce fronteras, cada empresa debe ser transnacional en su concepción, pero las multinacionales actuales también podrían volverse obsoletas, fabrican y distribuyen en el extranjero, pero en cada país son empresas locales. Con el comercio electrónico, estas empresas ya no son ni extranjeras, ni locales, «dónde fabricar o cómo vender ya no serán preguntas esenciales, no determinarán lo que hace una empresa, cómo lo hace y dónde lo hace» (Drucker, 2000).

\section{ECONOMÍA DIGITAL}

La incorporación de las tecnologías de la información y de las comunicaciones en la empresa en particular y en la sociedad en general, está influyendo de forma significativa en el entorno competitivo actual. Tal es la influencia de estas tecnologías sobre la economía, que desde algunos ámbitos se considera que se está presenciando la aparición de una nueva economía denominada «Economía Digital».

Debe reconocerse que las NTIC, están cambiando profundamente la manera de actuar de algunas empresas y sectores; sin embargo, el término de Nueva Economía no es adecuado. De hecho, cuando analizamos estudios sobre la nueva economía, descubrimos que dichos estudios se apoyan en los mismos paradigmas, los mismos conceptos y las mismas teorías que los estudios de la «Vieja Economía», quizás este haya sido el gran error cometido por muchas empresas, pensar que los nuevos modelos de negocio estaban fundamentados en otros pilares. La ley de la oferta y la demanda sigue siendo aplicable a los mercados electrónicos, la cadena del valor de Porter (2001), sigue siendo un marco válido para analizar los procesos de creación de valor de las empresas punto com, las empresas en Internet siguen utilizando el Marketing Mix como base de la estrategias de marketing, la técnica de flujos libres de caja, es una de las más utilizadas a la hora de realizar la valoración de empresas de la nueva economía, entre otras.
«Los paradigmas, los conceptos y las teorías que se utilizan en el estudio de la Nueva Economía, aparentemente son los mismos que los que se utilizan en la Vieja Economía, la novedad radica en las nuevas aplicaciones, matizaciones y desarrollos del cuerpo teórico tradicional» (López \& Sandulli, 2007). No obstante, se respetará el concepto cuando sea manejado por otros autores.

\section{DIFERENTES CLASIFICACIONES DEL COMERCIO ELECTRÓNICO}

Es importante conocer las diferentes clasificaciones que se pueden dar al hablar de comercio electrónico, cuando el empleo de Internet en las empresas era escaso, ya las primeras clasificaciones de sus posibles empleos cubrían la práctica totalidad de actuaciones de las mismas. Por ejemplo, en 1996, el profesor Oliver Hance hace la siguiente clasificación (Hance, 1996 citado en Oltra, 2004):

1. Internet para transacciones comerciales.

a) Internet para publicidad y mercadotecnia.

b) Internet en el comercio.

c) Internet para hacer y recibir pagos.

2. Internet y la comunicación (interna y externa).

3. Internet para investigaciones, desarrollo e intercambio de información profesional.

4. Internet para recursos humanos.

Sibien, la referencia viene desde 1996, esta clasificación sigue siendo válida aun en nuestros días.

Es con el uso mayoritario de la www y la interactividad que ésta supone, cuando la presencia electrónica de las empresas se convierte en una realidad incontestable, provocando el mayor número de estudios en los autores «clásicos» de trabajos de marketing, Kotler (citado en Oltra, 2004), habla del sitio web de empresa como algo diseñado para mantener una comunicación iniciada por el consumidor... y llega a diferenciarlo del «sitio web de marketing», que es diseñado para atraer a los clientes potenciales y actuales más cerca de una compra.

Se hace precisa una clasificación más exhaustiva, pero ¿según qué criterios?, en la tabla I se observan los más extendidos, aunque dejando sentado a priori que el primero de ellos, la clasificación según los agentes que intervienen es el mayoritariamente empleado, tanto por la prensa científica y la empresarial, como por la profana (Oltra, 2004). 
TABLA I. CLASIFICACIÓN.

Clasificación

\begin{tabular}{ll}
\hline Según los & Dentro del comercio electrónico, se puede elaborar una \\
agentes & clasificación muy prolija; dependiendo de los agentes que \\
que & intervien en en el proceso de comercio electrónico se \\
intervienen & pueden distinguir una gran variedad detipos. \\
& La nomenclatura con que se denominan responde a la \\
& siguiente sintaxis: [letra]2[etra], siendo [letra] la \\
& denominación de un agente, y el guarismo "2", una \\
& abreviación visual del artículo sajón "to". Así pues B2C \\
& debería leerse "Business to Consumer", relacionando \\
& pues "Empresas" con "consumidores". Rara vez se \\
& distinguen las relaciones como unidireccionales (Gil \\
& Estallo, 2001: 124 citado en Oltra, 2004), entendiéndose \\
& en nuestro trabajo entonces que, por ejemplo, B2C es
\end{tabular}
Sinónimo de C2B. en nuestro trabajo entonces que, por ejemplo, $\mathrm{B} 2 \mathrm{C}$

Tipos

Comercio electrónico entre empresas (Business to business; B2B)

Comercio electrónico entre empresa y consumidoı (comercio minorista; business to consumer; B2C)

Estas dos categorías son las consideradas de manera clásica como mínimo. Pueden darse muchas opciones en este tipo de clasificaciones de comercio electrónico.

- Comercio en tre empresas y gobierno en Internet. (B2G)

Relación en tre ciudadanos y gobierno en Internet. (G2C)

Comercio en tre consumidores (C2C).

- De amigo a amigo (P2P) "Peer to Peer".

- Relación o comercio entre la empresa y el empleado (comunicación entre empresa y trabajador; Business to Employee (B2E)

- Entre gobierno(G2G)

- Empresa y sociedad (B2S) Web social

- Educación y ciudadanos (E2C) e-learning

Según la Ya desde muy temprano, fue esta una posibilidad elegida tipología de a la hora de clasificar el comercio en Internet. Una primera senvicios comerciale s ofrecidos clasificación muy temprana (y muy elemental) nos la da Kehoe (Kehoe, 1994: 87- 92 citado en Oltra, 2004)

Más adelante se identifican por un buen número de • Bienes y servicios digitales autores (Águila; Padilla; Serarols; Veciana, 2001: 11-12 citado en Oltra, 2004), (Margherio, 1998; Kling, 1990; 2000; Kling y Lamb, 1999 citado en Oltra, 2004) cuatro subsectores en la economía digital.

Bienes y servicios digitales mixtos

- Producción de bienes y servicios intensivos en TIC

La ind ustria de las TIC que da soporte a los tres segmentos anteriores

Según la El concepto de "tecnología" como clasificador de los tecnología medios por los que puede ser realizado el comercio utilizada electrónico es empleado por los autores de manera muy diversa. La más elemental de ellas es la clasificación que hace la profesora del Águila (Águila, 2000a: 39); (Águila, 2000b: 59 citado en Oltra, 2004). Del Águila establece la distinción más básica: se emplea In ternet o no.

Escobar (Escobar, 2000: 27) plantea otra clasificación centrándose en la tecnología que se ofrece a través del medio.

- Comercio electrónico basado en In ternet

Comercio electrónico tradicional

Aplicaciones del comercio electrónico en el mercado cerrado

- Aplicaciones del comercio electrónico en el mercado abierto - Infraestructura de servicios

- Infraestructura de distribución de men sajería e in formación

- Infraestructura de contenidos multimedia y redes de documentos e información

- Infraestructura de red

Una de las clasificaciones sobre la base de la tecnología más extendidas hoy es la que se centra en la posibilidad de hacer negocio a través de los dispositivos con capacidad de proceso, que cada vez son más y más variados. Una buena clasificación de éstos puede ser la ofrecida por López (López, 2002: 61 citado en Oltra, 2004).

Grado de Nuevamente se puede presentar un conflicto al hablar de complejidad "complejidad". ¿A que nos referimos? ¿A la complejidad del negocio o la de las herramientas empleadas en él? La primera opción es la elegida por la profesora del Águila (Águila, 2000a: 39 citado en Oltra, 2004), quien nos da una clasificación muy simple: actividades poco complejas (pre/post venta, presencia electrónica...) o actividades complejas (venta de productos y servicios, pagos electrónicos, etc. Este mismo criterio lo emplean también González, Laguna y Mercado (González; Laguna; Mercado, 2000: 463-464 citad o en Oltra, 2004)

Para nuestro estudio, el conocimien to de las herramientas es fundamental. Y de entre ellas, la web destaca por ser hoy por hoy la herramienta estrella dentro del comercio electrónico. Un estudio exhaustivo de la complejidad de la misma lo ofrece Olsina en el capítulo segundo de su tesis doctoral, a quien seguimos casi de forma literal (Olsina, 1999 citado en Oltra, 2004).

Olsina establece una clasificación de Webs, considerando rangos de complejidad (estáticas versus dinámicas), y grados de orientación (orientado a documentos versus orientado a aplicaciones).
- Ordenadorconvencional

Webphones

PDA

Teléfonos móviles: Wap, SMS (e-commerce o Comercio Electrónico móvil, hoy tan de moda gracias a los programas televisivos)

- Web TV o TVCommerce (hoy no tan difundidos. EI PPV puede ser una variante del mismo)

- Solo estático

Sitio estático con formularios de en trada

Sitio con acceso de datos dinámicos

Aplicación de software basada en la Web
Sitio creado dinámicamente

Fuente: Oltra, 2004. 


\section{ASPECTOS IMPORTANTES DEL COMERCIO ELECTRÓNICO}

Existen ciertas situaciones sobre el comercio electrónico en relación a la empresa del que se escribe poco, a continuación se detallan algunos:

\section{Alianzas estratégicas entre PYMES para la generación de negocios electrónicos.}

Una de las nuevas ventanas de oportunidad que se abre en el horizonte empresarial, es el del comercio, a través de la red Internet. La cooperación entre empresas se manifiesta como una posible solución, para que manteniendo la autonomía y flexibilidad que es propia a la PYME, pueda tener acceso a los recursos y capacidades necesarios para competir, que en muchas ocasiones son patrimonio exclusivo de las grandes corporaciones. En este sentido, las alianzas estratégicas y la cooperación entre PYMES, se muestran como una forma de obtener los recursos y capacidades necesarios para competir en la red Internet, desarrollando negocios electrónicos (Medina \& Bruque, 2004), en la red todas las empresas parecen del mismo tamaño.

Gracias a las alianzas, las PYMES pueden mantener su flexibilidad y su autonomía, acceder a los recursos y capacidades que necesitan y mejorar sus costos de producción y transacción.

Mantener su flexibilidad y su autonomía; los motivos que pueden llevar a forjar una alianza pueden ser (Paz, 2001 citado en Medina \& Bruque, 2004), obtener ventajas competitivas para hacer frente a la competencia, creciendo a pesar de tener pocos recursos; o bien, logrando el control de ciertos recursos, aprovechar las oportunidades con rapidez y con flexibilidad, lo que podría requerir recursos y capacidades de difícil desarrollo y adquisición que son accesibles a través de alianzas.

Acceder a los recursos y capacidades; la segunda perspectiva puede ser analizada desde una visión basada en recursos y capacidades de la empresa, a la que se le ha reconocido su potencial explicativo en el fenómeno de las alianzas (Gulati, Nohria y Zaheer, 2000 citado en Medina \& Bruque, 2004).

Las carencias de los recursos y capacidades necesarios es una de las más importantes debilidades de las PYMES que desean crear un negocio electrónico. Pero... ¿por qué no simplemente se desarrollan los recursos o capacidades que se requieren, se compran, o se adquiere una empresa que ya los posea?

En este contexto, las alianzas entre empresas que ponen en común sus mejores recursos y capacidades para competir de forma coordinada, cobran sentido.

Mejorar sus costos de producción y transacción; esta perspectiva puede ser estudiada bajo los principios de la economía de los costos de transacción (Williamson, 1985 y 1991; Powell, 1990; Thorelli, 1986; Jarillo, 1988 y 1993; McGee, 1999 citado en Medina \& Bruque, 2004), para la que existe un nuevo punto de equilibrio entre la jerarquía y el mercado, que hace más racional externalizar ciertas actividades (Jarillo, 1988, 1993 citado en Medina \& Bruque, 2004), ahora fácilmente controlables con las nuevas tecnologías de la información (Rockart y Short, 1989; Clemons y Row, 1992; Kumar y van Dissel, 1996 citado en Medina \& Bruque, 2004).

Las empresas no pueden tener los costos más bajos de su sector en todas las actividades que realizan dentro de sus límites organizativos. Las alianzas permiten la reducción de los costos de producción, ya que las empresas que intervienen en alianzas pueden concentrarse en aquello que saben hacer mejor (reduciendo costos al evolucionar por la curva de aprendizaje y al desarrollar economías de escala) y dejar la realización del resto de actividades a otras empresas que son las mejores y más baratas en su labor (McGee, 1999 citado en Medina \& Bruque, 2004).

Por otra parte, la realización de transacciones con otras empresas en el mercado abierto exige grandes esfuerzos (como son los costos) al tener que buscar empresas con las que comerciar, y tener que coordinarse con las mismas. Además, existe el 
riesgo de que las empresas con las que se realiza transacciones desarrollen conductas oportunistas y no respeten los acuerdos, o exploten en su beneficio la relación. No obstante, gracias a las alianzas estratégicas (que son estables y basadas en la confianza), y a las modernas tecnologías de la información (que permiten buscar fácilmente empresas con las que entablar relaciones, coordinarse con las mismas, y controlar sus comportamientos al reducir las asimetrías de información), es posible reducir los costos de realizar transacciones con otras empresas. En este sentido, se reducen los costos de búsqueda de otras empresas, se mejoran los costos de coordinación y se reduce la amenaza de comportamientos oportunistas por parte de las empresas con las que se realizan transacciones (Clemons y Row, 1992; Kumar y van Dissel, 1996 citado en Medina \& Bruque, 2004).

\section{ADOPCIÓN DEL COMERCIO ELECTRÓNICO}

De acuerdo al Diccionario de la Real Academia Española; adopción: es recibir, haciéndolos propios, pareceres, métodos, doctrinas, ideologías, modas, etc., que han sido creados por otras personas o comunidades, es lo que se intenta generar con el comercio electrónico, para una mayor penetración, que funcionaria mejor que una simple adaptación; sin embargo, existen algunas situaciones que se deben de tener en cuenta, como factores inhibidores, (Villanueva \& Iniesta, 2001) y aspectos culturales (Aqueveque \& Fernández, 2004).

Algunos factores inhibidores pueden ser:

Factor 1: Seguridad. Las variables con más peso en este factor son aquellas que hacen referencia a frenos relacionados con la seguridad del comercio en Internet o con la privacidad de los datos. Ambos aspectos muy relacionados, y que por tanto llamaremos «seguridad».

Factor 2: Desconfianza en el canal. En este factor vemos claramente tres variables que pesan mucho más fuertemente que el resto: «juzgar calidad», «vendedor no familiar», «falta información». Por tanto, este factor hace referencia a la desconfianza que algunas personas presentan hacia el canal (Internet).
Factor 3: Falta de oferta. En este tercer factor, dos son las variables más importantes: «no encuentra sitios adecuados», o "el sitio no ofrece la posibilidad de compra». Este factor, por tanto, afecta a aquellos que buscan productos o servicios que no están en Internet o que, si están, no son muy conocidos o fáciles de encontrar.

Factor 4: Estilo de vida. Las variables con mayor peso son: «más fácil localmente», "prefiere gente». Este factor muestra, por tanto, que algunos consumidores simplemente prefieren el comercio tradicional, en el que pueden tener un trato más personal interactuando con personas y que está más accesible localmente.

Factor 5: Muy complicado. Indica que algunas personas ven Internet como un canal demasiado complicado para ellos. Los pormenores últimos de esta aparente «complicación» no vienen especificados.

Factor 6: Falta de tarjeta de crédito. Indiscutiblemente, aquellas personas que no disponen de tarjeta de crédito tienen el acceso vedado a gran parte de los sitios de venta por Internet.

Factor 7: Política de la empresa. Algunas empresas prohíben a sus empleados utilizar Internet para realizar compras. De nuevo, sería interesante conocer más detalles por los que estas políticas vienen a establecerse en el caso de compras de empresa.

Factor 8: Mala experiencia. Por último, el octavo factor indica claramente que hay gente que ha probado, ha tenido una mala experiencia, y esto le ha llevado a no comprar más. Este factor es uno de los más controlables por parte de la industria.

Como puede observarse, los factores anteriores son inhibidores del proceso de adopción del comercio electrónico, también es cierto que ninguno de ellos son insalvables, con una correcta gestión sobre el nuevo canal de compra. 


\section{Cuestiones culturales}

Las características culturales de un país afectan el desarrollo del comercio electrónico, con niveles de impacto iguales o superiores a los factores económicos, de mercado y tecnológicos considerados hasta el momento.

El estado de desarrollo tecnológico, económico, educativo y de penetración de Internet en los hogares, es el principal determinante en las posibilidades de desarrollo de comercio electrónico en un país. Esta conclusión es coherente con la mayoría de las visiones previas, obtenidas a partir de estudios internacionales; por lo tanto, el gran desafío futuro es disminuir la «División Digital», y así permitir a aquellos países más atrasados en el campo tecnológico acceder a la Nueva Economía.

La importancia que una cultura o sociedad le tenga al materialismo, determinará sus patrones de consumo. El sentido de posesión y el valor asociado a ella son factores importantes en culturas «masculinas», por lo que se puede esperar un mayor nivel de consumo, si a esto se le suma la característica de «exclusividad» que puede adquirir un bien al ser comprado a través de Internet, se podría estar frente a la explicación de la importancia de esta variable en el desarrollo de este tipo de comercio.

La urbanidad de una cultura, medida como el porcentaje de la población que vive en áreas urbanas, y su grado de conexión a Internet y a la televisión por cable también determina o condiciona las posibilidades de desarrollo del comercio electrónico.

Lavariable de distancia del poder, referida principalmente al grado de aceptación de las desigualdades en la distribución del poder, pareciera tener un efecto que se debe considerar, o por lo menos estudiar, aunque la explicación de esto aún no es clara y debería ser objeto de un estudio más profundo y de tipo antropológico, que permita relacionar este valor cultural con los patrones de consumo de los individuos.
El hecho de que casi el $90 \%$ de los servidores seguros estén asociados a páginas o sitios en idioma inglés, ,condiciona las posibilidades de realizar transacciones de los individuos, lo cual disminuye considerablemente las posibilidades de transacciones seguras para los habitantes de países con idiomas distintos a éste; sin embargo, parece no ser un factor relevante, lo que se podría explicar debido a que si bien hay países en los cuales la lengua madre no es el inglés (europeos y asiáticos principalmente), gran parte de la población domina este idioma.

La variable cultural individualismo parece tener un efecto negativo sobre el comercio electrónico, aunque esta afirmación es cuestionable dado algunos antecedentes por analizar. Según el modelo, mientras más individualista sea un país, menor será el grado de comercio electrónico; sin embargo, Estados Unidos es el país más individualista del mundo y es, después de Japón, el país con mayor volumen de transacciones de comercio electrónico por usuario. Esto entrega una evidencia que indica que esta relación debe ser analizada con mayor detención.

La juventud de un país pareciera tener algún grado de efecto sobre la potencialidad de desarrollo del comercio electrónico, aunque en el caso de la muestra no es tan claro. Tradicionalmente se considera que son los jóvenes quienes más navegan y adquieren productos a través de la red, por lo que países de mayor juventud deberían presentar índices de comercio electrónico mayores, sin embargo, esto no es tan claro y se podría explicar en el hecho de que aquellos países desarrollados, en los que se tiene más recursos, poder adquisitivo, acceso a la red y, por lo tanto, comercio electrónico, son normalmente más «viejos» que aquellos en vías de desarrollo (Aqueveque \& Fernández, 2004).

El resto de las variables; es decir, prevención de la incertidumbre, costo de acceso y proveedores de servicio y seguridad, parecen ser irrelevantes en la muestra considerada, desde el punto de vista de la 
variable dependiente, la explicación para esto puede depender de cada una de las variables. En el caso del costo de conexión, los antecedentes recopilados indican que siempre ha sido considerado como relevante; asimismo resultado para esta muestra de países se podría explicar dado la homogeneidad de ella en estas variable; es decir, este resultado particular se puede deber a que en el caso de la muestra, no se presentan grandes diferencias desde el punto de vista de los costos de conexión y considerar esta variable al momento de analizar países con un menor grado de desarrollo, ya que en esos casos pudiera ser fuente de influencia considerable. Para el caso de los proveedores de servicio y seguridad, la explicación de su bajo nivel de incidencia pudiese ser la misma, aunque el tema del número de servidores seguros realmente diferencia a los países; sin embargo, si se considera que la mayor cantidad de transacciones se realizan en sitios de tipo internacional, esta variable pierde relevancia. El tema de la prevención de incertidumbre debería ser analizado, ya que se considera que en las primeras etapas de desarrollo del comercio electrónico, el factor riesgo percibido puede ser de importancia(Quelch, 1996 citado en Aqueveque \& Fernández, 2004), no sólo en el corto plazo, sino que también en el largo plazo es la barrera de mayor importancia en el desarrollo del potencial de mercado de Internet (Keen, 1997 citado en Aqueveque \& Fernández, 2004). Un estudio sobre la relación de esta variable cultural con las percepciones de riesgo podría explicar estos resultados.

\section{CONCLUSIONES}

El comercio electrónico como esquema con uso intensivo de tecnología, debería de entenderse como un fin para hacer la vida más cómoda y como herramienta necesaria en un mundo vertiginoso, donde el ahorro de tiempo y reducción de costos es necesario. No viene a desplazar a nada, simplemente es una forma más, un canal más, el cual es necesario que las empresas conozcan y administren correctamente, que en la mayoría de los casos es subutilizado en forma de un simple portal de información y correo electrónico. Se debe de aprovechar todo su potencial, formularios con información valiosa de los clientes, que se puede incluir en un sistema de administración basada en la relación con los clientes (CRM), Customer Relationship Management aprovechando la información digitalizada

MAYO-AGOSTO 2012. Año 18, Número 51. para manejar grandes volúmenes de ésta, dar la mejor imagen de sí; en el ciberespacio no importa el tamaño de la empresa, grande, mediana, pequeña, todas se parecen iguales. Por el lado del cliente, se debe de buscar una mayor adopción por parte de ellos, considerar las barreras sociales, culturales y económicas, para acelerar dicha adopción; entender una relación de ganar/ganar, el cliente gana en precio, comodidad, rapidez, atención y la empresa gana en efectividad, eficiencia y eficacia. La seguridad de la red es responsabilidad de todas aquellas empresas que requieran usar este canal, por lo que cada vez habrán mejoras en ese aspecto, hay que ser cautos con las expectativas no se debe caer en los errores especulativos que generaron la burbuja de las empresas punto como a finales de los noventa con el consabido boom, no se trata de «subirnos» al cambio tecnológico, ser parte de él, debidamente preparados, con las bases organizacionales que permitan el sostenimiento del negocio.

\section{HITOS DE CIENCIAS ECONOMICO ADMINISTRATIVAS}

www.ujat.mx/publicacions/hitos 
REFERENCIAS

Aqueveque, C. \& Fernández, C. (2004). El efecto de las características culturales de un país en la adopción del Comercio Electrónico B2C. Pensamiento \& gestión, (17). Universidad del Norte, 39-64.

Castells, M. (1997). La era de la información. Ed. Alianz Editorial, Madrid.

Conklin, D. W. \& Tapp, L. (2000). The creative Web: a new model for managing innovation. Ivey Business Journa, volumen 64(5), 60-68.

De la Guardia, C. (2001). La evolución del Comercio Electrónico. Revista Razón y Palabra. Noviembre 2000-Enero 2001. (20). Recuperado de http://www.razonypalabra.org. $\mathrm{mx} /$ anteriores/n20/20_cguardia.html

Drucker, P. F. (2000).Gutenberg, la máquina de vapor, Internet... y más allá. Quark: Ciencia, medicina, comunicación y cultura, (18), 25-31.

Gimeno, M. (2006). Las tecnologías de la información y la comunicación en las PYMES y otros avatares. Revista de Economía Industrial, (360), 77-92.

Hofer, C. W. \& Bygrave, W. D. (1992). Researching Entrepreneurship. Journal Entrepreneurship Theory and Practice, volumen 16(3), 91-100.

Jaramillo, O. (1986). Las nuevas tecnologías de la información y el ejercicio profesional. Memorias del VEncuentro Latinoamericano de Facultades de Comunicación Social, Felafacs. Bogotá.

López, J. I. \& Sandulli, F.D. (2007). Evolución de los modelos de negocios en Internet: situación actual en España de la economía digital. Revista Economía Industrial, (364).Universidad Complutense de Madrid. 213-229.

Medina, J. A. \& Bruque, S. (2004). Las alianzas estratégicas entre pymes para crear negocios electrónicos. Revista: Futuros, volumen 2(5). Recuperado de http://www.revistafuturos.info/

\section{futuros_5/pyme_1.htm.}

Moliní, F. (2002). Ventajas, inconvenientes e impactos territoriales del Comercio Electrónico. Investigaciones Geográficas, (27), 131-150

Muñoz, J. M. (2008). «NNTT, TIC, NTIC, TAC... en educación ¿pero esto qué es?». Quaderns digitals: Revista de Nuevas Tecnologías y Sociedad, (51), 1-9.

Oltra, J. V. (2004). Clasificaciones del Comercio electrónico. Departamento de Organización de Empresas, Economía Financieray Contabilidad, Universidad Politécnica de Valencia.

Padilla, A. \& Serarols i Tarres, C. (2006). Las características del empresario y el éxito de las empresas puramente digitales. Tribuna de Economía. Noviembre-Diciembre, (833), 155-176.

Pardo Bustillo, F. (2004). El Comercio Electrónico en España hoy: Aspectos cuantitativos y cualitativos. El Comercio en la SI, (813), 27-42

Porter, M. (2001). Strategy and The Internet. Harvard Business Review, volumen 79(3), 63-78.

Poster, M. (1995). The Net as a public sphere? Wired, volumen 3(11), 136-137

Villanueva, J. \& Iniesta, F. (2001). Factores inhibidores en la adopción de Internet. Economía Industrial, volumen 4(340), 93-100 